\title{
Utilidad de la estimulación continua del núcleo pedunculopontino para el tratamiento de la enfermedad de Parkinson
}

\section{Utility Continuous Stimulation of Pedunculopontine Nucleus for treating Parkinson's Disease}

Fecha de recepción: 14 Octubre 2015 | Fecha de aprobación: 15 Marzo 2017

\author{
Stephany Barbosa Balaguera \\ Pontificia Universidad Javeriana, Colombia \\ Miguel Germán Borda \\ Pontificia Universidad Javeriana, Colombia \\ Óscar Zorro \\ Hospital Universitario San Ignacio, Colombia \\ Juan Carlos Acevedo González \\ Pontificia Universidad Javeriana, Colombia
}

a Autor de correspondencia. Correo electrónico: jacevedog@gmail.com

Cómo citar: Barbosa Balaguera S, Borda MG, Zorro O, Acevedo González JC. Utilidad de la estimulación continua del núcleo pedunculopontino para el tratamiento de la enfermedad de Parkinson. Univ Med. 2017;58(3):1-5. doi: https://doi.org/10.11144 / Javeriana.umed58-3.park

\section{RESUMEN}

El núcleo pedunculopontino contiene gran cantidad de conexiones que modulan la actividad motora en los humanos; por este motivo, se ha planteado que su estimulación profunda tendría beneficios significativos en el tratamiento de la enfermedad de Parkinson. Con una carga orgánica y social significativa, la enfermedad de Parkinson reúne una serie de signos y síntomas, principalmente motores, que afectan significativamente la calidad de vida de los pacientes que la padecen. Actualmente, se encuentran dentro de un área de investigación con gran potencial para dar manejo a los síntomas de esta enfermedad, y se desconoce si su estimulación cerebral profunda podría orientar futuras intervenciones con resultados óptimos. Por esta razón, la revisión busca esclarecer la utilidad de este procedimiento; sin embargo, es bastante controvertido y su evidencia escasa, además de que es difícil centrarse únicamente en un núcleo para resolver los problemas relacionados con dicha enfermedad.

Palabras clave

órganos blanco; estimulación cerebral profunda; enfermedad de Parkinson.

\begin{abstract}
The pendunculopontine nucleus contains many connections responsible of modulate motor activity. It has been suggested that deep stimulation would have significant benefits in the treatment of Parkinson's disease, intervention that could improve the patient's quality of life and generate a positive impact in public health due Parkinson's disease has important organic and social burden. There is a growing area of research in this field, however is still uncertain if deep brain stimulation could guide future interventions with optimal results. For this reason, we pretend to clarify the existing knowledge of this procedure, nevertheless, it is quite controversial, we consider that it is difficult to focusing on a unique nucleus to solve the problems associated with this disease.

Keywords

pedunculopontine nucleus; deep brain stimulation; Parkinson`s disease.
\end{abstract}




\section{Introducción}

La enfermedad de Parkinson (EP) es un trastorno neurodegenerativo generalizado [1] responsable de un síndrome motor, secundario principalmente a la denervación dopaminérgica nigroestriada y acompañado de una alteración colinérgica [2]. Se trata de una enfermedad con una carga orgánica y social significativa que conduce a la disminución del autocuidado, jubilación anticipada y dependencia física, siendo más allá de eso un problema de salud pública [3]. En la actualidad, a pesar de los tratamientos farmacológicos, es una enfermedad de difícil manejo y, por tal motivo, se han planteado nuevos tratamientos que tienen como objetivo principal modular la actividad neuronal anormal y aliviar los síntomas [4]. Esta revisión no sistematizada de la literatura está centrada en la estimulación de núcleo pedunculopontino (NPP) como estrategia terapéutica para el manejo de la enfermedad de Parkinson, destacando aspectos importantes de la biología y la relación con dicha terapia.

\section{Núcleo pedunculopontino: localización y funciones}

El NPP, descrito inicialmente por Jacobson, a principios del siglo $\mathrm{XX}$, se encuentra anatómicamente ubicado en el área tegmental ventral y corresponde al grupo Ch5 de Mesulam (nomenclatura descrita por Mesulam, en 1983, utilizada para describir las vías colinérgicas ascendentes y los principales núcleos colinérgicos; sus límites se describieron por Rye y Saper, basados en experimentos con ratas) [5]. Está conformado por neuronas colinérgicas (pars compacta) y no colinérgicas (pars dissipata). Posee gran cantidad de conexiones aferentes y eferentes con los ganglios basales, tálamo, protuberancia, bulbo, médula espinal, cortezas sensorio-motoras, cerebelo, entre otros [6]. Dichas conexiones le permiten intervenir en la regulación del ciclo vigiliasueño, control de locomoción y posiblemente es un centro de integración de la información motora. Adicionalmente, se ha comprobado que tendría implicaciones importantes en enfermedades del sistema nervioso central como: parálisis supranuclear progresiva, narcolepsia, esquizofrenia $y$, sobre todo, enfermedad de Parkinson [5].

Existe una distribución topográfica de la actividad neuronal del NPP en sus regiones caudal y rostral, que coinciden con los datos que existen actualmente descubiertos en animales y que confirman una particular relevancia en el control de la marcha [6]. Se considera un centro integrador de los impulsos motores que se encarga de transformar la motivación en acción o comportamiento. Recibe información de los ganglios basales sobre el control postural y puede modular centros medulares o bulbares implicados en la locomoción [5].

\section{Relación fisiopatoloógica entre el núcleo pedunculopontino y la enfermedad de Parkinson}

La EP se caracteriza por una pérdida de neuronas dopaminérgicas que generan el cuadro clínico característico: bradicinesia, rigidez, inestabilidad postural, temblor y alteración en la marcha [3]. Sin embargo, estudios histológicos de tejido cerebral de pacientes con EP idiopática o secundaria a tóxicos (hidrocarburos) han encontrado una pérdida significativa de neuronas y cuerpos de Lewy en el NPP [5]. Igualmente, se identificó una pérdida de la influencia excitatoria pontina sobre la sustancia nigra pars compacta, hacia donde terminan los axones colinérgicos del NPP que se asocia a degeneración temprana del núcleo [2].

Los cambios microestructurales del NPP y de las estructuras subcorticales están directamente relacionados con la sintomatología de los pacientes con EP [7]. Se ha descrito que en esta enfermedad existe una disfunción en las vías eferentes que se dirigen desde el NPP hacia los ganglios basales; esto genera hipoactividad del área motora mesencefálica y del propio NPP [2]. El daño de la inervación colinérgica del tálamo que proviene del NPP, principalmente, cumple un 
papel importante en el temblor característico de la EP [5].

\section{Tratamiento de la enfermedad de Parkinson con estimulación continua del núcleo pedunculopontino}

Los primeros reportes científicos sobre estimulación cerebral de NPP a baja frecuencia son del 2005 [5]. Este hallazgo planteó una nueva alternativa de tratamiento en EP, al promover su estudio, sobre todo en marcha e inestabilidad postural [4].

La estimulación cerebral profunda (ECP), principalmente en el globo pálido, pars interna y núcleo subtalámico, se ha utilizado como terapia en pacientes con EP previamente seleccionados que presenten fluctuaciones motoras, discinesias inducidas por medicación y temblor refractario debilitante, con resultados favorables [4]. También, la estimulación de NPP ha dado resultados favorables en las etapas tardías de la EP, sobre todo en los signos axiales como trastornos posturales y de la marcha, que generalmente son refractarios a otros tratamientos [2].

Un estudio realizado por Rauch y colaboradores, en el 2010 [8], en el que utilizaron ratas macho Sprague-Dawley, les administró 6-hidroxidopamina en el equivalente humano del NPP para causar un daño tóxico e inducir hemiparkinsonismo (modelo experimental de creación de enfermedad de Parkinson en animales) para posteriormente someterlos a ECP durante 24 horas con diferentes frecuencias. Posteriormente, utilizando un cilindro transparente de $26 \mathrm{~cm} \times 29 \mathrm{~cm}$ durante $5 \mathrm{~min}$, observaron el empleo de la extremidad anterior izquierda con la contralateral durante el ejercicio. En la prueba de eliminación de adhesivo, en 5 oportunidades pusieron cinta adhesiva en la nariz de la rata y evaluaron el uso de su extremidad anterior izquierda y derecha para retirarla. En la prueba de campo abierto: dejaron la rata en una pista de movimiento durante 20 min y calcularon el tiempo total de movimiento. Por último, en la prueba de polos, muy sensible a disfunción nigroestriada, usaron una jaula envuelta en tela áspera y colocaron la rata con la cabeza hacia arriba cerca de la parte superior y calcularon 3 veces el tiempo que tomó la rata en girar y bajar. Determinaron que con estimulación a baja frecuencia (25 $\mathrm{Hz}$ ) se encontraban resultados favorables en el desarrollo de estas pruebas realizadas por las ratas, lo cual podría servir eventualmente como tratamiento de trastornos de la marcha y la inestabilidad postural en EP [8].

Saryyeva y colaboradores [9], en el 2011, midieron la expresión de c-Fos (marcador de actividad neuronal aguda) en el mismo modelo de ratas con previa intervención con 6-hidroxidopamina, utilizando diferentes frecuencias y en diferentes localizaciones (dorsal, medial ventral y ganglios basales), y afirmaron que el NPP podría llegar a ser un objetivo potencial en el tratamiento de la EP, sobre todo en la bradicinesia y rigidez severa e inestabilidad postural con frecuencia baja de $25 \mathrm{~Hz}$.

Diversos estudios han mostrado que la lesión excitotóxica unilateral del NPP en primates no humanos ocasiona una reducción en la influencia excitatoria de la actividad tálamocortical; por otro lado, este mismo procedimiento utilizado de manera bilateral genera un cuadro de inestabilidad postural y severos trastornos de la marcha que no corrigen al administrar agonistas dopaminérgicos, lo cual podría explicar los síntomas axiales observados en la EP, que no responden a tratamiento dopaminérgico $[2,10]$.

En un estudio prospectivo, en el que se estimuló la zona del NPP en pacientes con EP, se obtuvieron resultados favorables en una pequeña cantidad de pacientes, y describieron lo que podría ser una interacción negativa entre la ECP y el uso de levodopa, tanto antes como después de la intervención; adicionalmente, describió problemas del habla en los pacientes tratados, principalmente disartria e inteligibilidad (capacidad de ser entendido) [11]. Estudios realizados en ratas y gatos demuestran que la lesión del NPP causa aquinesia y que su estimulación tiene algunos efectos positivos motores [12]. Ello sugeriría que hay lesión directa del NPP en la EP. 
A pesar de que muchos estudios realizados en animales sugieren que la ECP del NPP podría ofrecer una mejoría en la marcha, no se ha establecido claramente su eficacia y sus efectos precisos en el tratamiento habitual de la EP [13]. Además, estudios en roedores y primates no humanos han revelado que la evolución de la locomoción cuadrúpeda a la bípeda no afectó la anatomía de las estructuras encargadas, sino que generó una distribución diferente en los circuitos y en la proporción neuronal [2]. Esto, tal vez, explicaría las diferencias que existen en la estimulación de NPP en animales, comparado con humanos.

Muchos autores sugieren que la clave del tratamiento radica principalmente en la escogencia selectiva del paciente, teniendo especial cuidado en las expectativas de los resultados y considerando las características de inclusión: inicialmente que sea una enfermedad de menos de 5 años de evolución, que exista respuesta dopaminérgica, que las escalas de demencia estén en rangos bajos, una imagen de resonancia magnética en $\mathrm{T} 2$ normal, pacientes preferiblemente menores de 69 años, y también sus contraindicaciones: EP atípica, enfermedades cardiovasculares, atrofias severas, alteraciones en el estado del ánimo, memoria y alteraciones psicóticas [14].

Existen mejores resultados en la ECP del NPP en comparación con la ECP del núcleo subtalámico, con respecto a los trastornos de la marcha y los episodios de rigidez presentes en la EP $[15,16]$. Adicionalmente, se sugiere una mejoría en los trastornos relacionados con el sueño, que son característicos en los pacientes con EP, ya que como se mencionó el NPP regula el ciclo sueño-vigilia [10]. Y se considera que en la EP existe una relación con el desequilibrio entre las frecuencias bajas y altas del NPP, lo cual confirmaría un papel importante en la regulación de la marcha [17].

\section{Conclusiones}

La ECP del NPP ha generado un nuevo campo de estudio, dado que tiene gran potencial como tratamiento en la EP; sin embargo, su aplicación clínica es bastante controvertida, y su evidencia, escasa. Estudios en animales sugieren que podría considerarse una opción terapéutica, pero las intervenciones que se han realizado en pacientes demuestran efectos secundarios de gran importancia.

Podría tratarse de la necesidad de una escogencia selectiva de los candidatos a ECP o puede existir una diferencia significativa entre el NPP en animales y en humanos o, tal vez, dado que la EP tiene una afectación generalizada, resultaría difícil centrarse en un solo núcleo para dar manejo completo e integral.

Se necesitan mayores estudios que contribuyan a determinar con exactitud la eficacia y la seguridad de esta nueva opción de tratamiento.

\section{Referencias}

1. Broeder S, Nackaerts E, Heremaans E, et al. Transcranial direct current stimulation in Parkinson's disease: Neurophysiological mechanisms and behavioral effects. Neurosci Biobehav Rev. 2015;57:105-17.

2. Blanco L, Lorigados L, Serrano T, et al. Acta biológica colombiana 2012;17:445-62.

3. Jankovic J. Parkinson's disease: Clinical features and diagnosis. Journal neurol. Neurosurg. Psychiatry. 2008;79:368-76.

4. Martinez-Ramírez D, Hu W, Bona A, et al. Update on deep brain stimulation in Parkinson's disease. Transl Neurodegener. 2015;4:12. doi: 10.1186/ s40035-015-0034-0

5. Erro E, Giménez-Amaya JM. El núcleo tegmental pedunculopontino: anatomía, consideraciones funcionales e implicaciones fisiopatológicas. Anal Sis San Navarra. 1999;22:189-201.

6. Thevathasan W, Pogosyan A, Hyam J, et al. Alpha oscillations in the pedunculopontine nucleus correlate with gait performance in parkinsonism. Brain. 2012;135:148-60. doi: 10.1093/brain/awr315 
7. Youn J, Lee J, Kwon $\mathrm{H}$, et al. Alterations of mean diffusivity of pedunculopontine nucleus pathway in Parkinson's disease patients with freezing of gait. Parkinsonism Relat Disord. 2015;21:12-7.

8. Rauch F, Schwabe K, Krauss J. Effect of deep brain stimulation in the pedunculopontine nucleus on motor function in the rat 6 hydroxydopamine Parkinson model. Behav Brain Res. 2010;210:46-53.

9. Saryyeva A, Nakamura M, Krauss J, Schwabe K. C-Fos expression after deep brain stimulation of the pedunculopontine tegmental nucleus in the rat 6hydroxydopamine Parkinson model. J Chem Neuroanat. 2011;42(3):210-7.

10. Belaid H, Adrien J, Lafrat E, et al. Sleep disorders in parkinsonian macaques: Effects of L-dopa treatment ande pedunculopontine nucleus lesión. J Neurosci. 2014;27:9124-33.

11. Pinto A, Ferraye $M$, Espesser $R$, et al. Stimulation of the pedunculopontine nucleus area in Parkinson's disease: Effects on speech and intelligibility. Brain. 2014;137:2759-72. doi: 10.1093/ brain/awu209

12. Nandi D, Liu X, Winter JL, et al. Deep brain stimulation of the pedunculopontine región in the normal non-human primate. J Clin Neurosci. 2002;2:170-4. doi: 10.1054/ jocn.2001.0943

13. Thevathasan W, Cole MH, Grapel $\mathrm{CL}$, et al. A spatiotemporal analysis of gait freezing and the impact of pedunculopontine nucleus stimulation. Brain. 2012;135:1446-54. doi: 101093/ brain/aws039

14. Martínez-Hernández HR, Arellano-Reynoso A. Estimulación cerebral profunda en enfermedad de Parkinson: primer caso tratado en el Centro Neurológico ABC. An Med Asoc Med Hosp ABC. 2012;57(1):53-61.

15. Liu $H$, Zhang $K$, Yang $A$, et al. Deep brain stimulation of the subthalamic and pedunculopontine nucleus in a patient with
Parkinson's disease. J Korean Neurosurg Soc. 2015;57:303-6.

16. Khan S, Gill SS, White P, et al. Combined pedunculopontinesubthalamic stimulation in Parkinson disease. Neurology. 2012;78:1090-5.

17. Fraix V, Bastin J, David $O$, et al. Pedunculopontine nucleus área oscillations during stance, stepping and freezing in Parkinson's disease. PlosONE. 2013;8: 12. doi: 10.1371/journal.pone.0083919 